\title{
Arbeiten in der Pflege macht krank
}

\section{BKK legt Gesundheitsatlas 2017 vor: Gesundheitsförderung dringend nötig}

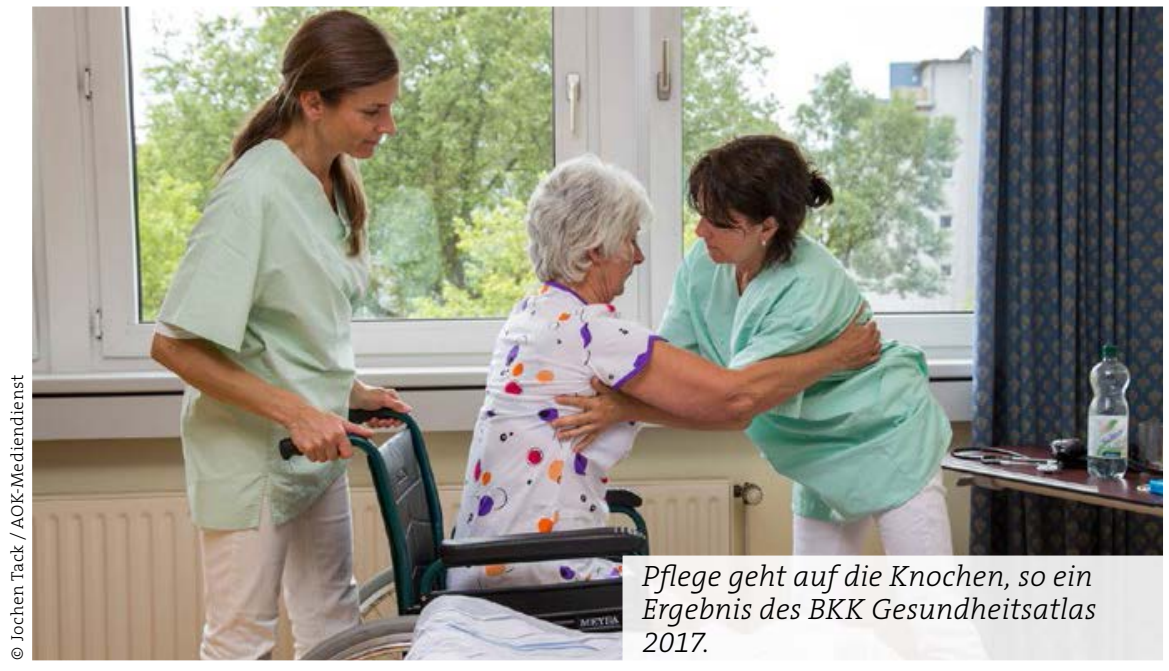

") Das Erkrankungsgeschehen in der Pflege ist besorgniserregend: Die dort Beschäftigten sind länger krank und haben mehr psychische Leiden. Das geht aus dem BKK Gesundheitsatlas 2017 hervor. Angemahnt wird, die betriebliche Gesundheitsförderung stärker zu nutzen.

Mehr als jeder zehnte Beschäftigte in Deutschland hat einen Gesundheitsberuf. Von diesen 3,2 Millionen sozialversicherungspflichtig Beschäftigten arbeitet nach Angaben der BKK nahezu die Hälfte in pflegerischen Berufen. Und die gehen offenbar ,auf die
Knochen“. Die Analysen belegen, dass „besorgniserregend viele Pflegekräfte aufgrund der Arbeitsbedingungen einen kritischen Gesundheitszustand“ haben. Dazu komme, dass jeder Dritte in der Altenpflege (32,9\%) lediglich ein befristetes Arbeitsverhältnis hätte, heißt es der BKK-Pressemitteilung. Die Daten basieren auf einer Umfrage im März 2017 unter 2.000 Beschäftigten verschiedener Branchen und Berufe zum Thema „Gesundheit und Arbeit“.

Vor allem die Gesundheit der weiblichen Beschäftigten scheint danach gefährdet. Im Vergleich zu allen ande- ren Beschäftigten, die im Schnitt 16 Tage krank waren, wurden hier deutlich längere Ausfallzeiten festgestellt: Rund 24 Tage sind es z.B. bei denjenigen, die in Pflege- oder Altenheimen arbeiten, im Krankenhaus waren es rund 18 Arbeitsunfähigkeitstage.

Spitzenreiter hinsichtlich Erkrankungen aufgrund psychischer Störungen sind ebenfalls weibliche Beschäftigte in Pflegeheimen: Sie sind doppelt so lange erkrankt wie der Durchschnitt aller Arbeitnehmer (4,6 Krankentage versus 2,3). Allerdings führen bei der stationären Behandlung aufgrund psychischer Diagnosen Männer in Pflegeberufen die Statistik an (15\% mehr als weibliche Beschäftigte). Dabei lägen alle Menschen, die in der Pflege arbeiten, ohnehin schon jeweils mehr als 55\% über dem Schnitt der Krankenhaustage aller Berufstätigen, die aufgrund psychischer Diagnosen im Krankenhaus waren, so die BKK.

Auch körperliche Beschwerden sind bei Beschäftigen in Pflegeberufen auffällig häufig. So fallen aufgrund von Muskel- und Skelettkrankheiten z.B. Altenpflegerinnen doppelt so lange aus wie die weiblichen Beschäftigten insgesamt (7,0 versus 3,7 Krankentage), wie die BKK berichtet.

(run)

www.bkk-dachverband.de

\section{Sicherheit mit Armbändern zur Patientenidentifikation}

\section{Stiftung Hospital zum Heiligen Geist: Schutz der Patienten an erster Stelle}

» Jeder Patient, der stationär im Hospital zum Heiligen Geist oder im Krankenhaus Nordwest in Frankfurt am Main aufgenommen wird, erhält seit dem 1. Juli 2017 ein Patientenidentifikationsarmband. Damit folgen die beiden Einrichtungen der Stiftung Hospital zum Heiligen Geist den Empfehlungen des bundesweiten Aktionsbündnisses Patientensicherheit.

Auf dem Armband aufgedruckt sind persönliche Daten wie der vollständige Name, das Geburtsdatum, die zuständige Station, die Fallnummer sowie ein Barcode. Diese Daten dienen der eindeu- tigen Identifikation der Patienten, z.B. bei der Medikamentenvergabe oder bei medizinischen Eingriffen. Gerade in Situationen, in denen Patienten selbst keine Auskunft geben können, etwa nach der Einnahme sedierender Medikamente oder in Narkose, bei Verständigungsproblemen, bei der Notwendigkeit zu schnellem medizinischen Handeln, greift das Armband als zusätzliche Sicherheitsmaßnahme, um Verwechslungen von Personen auszuschließen. Die Armbänder bestehen aus einem hypoallergischen, latexfreien Kunststoff, was die problemlose Reinigung und
Desinfektion ermöglicht. Sie vertragen alle Aktionen des Alltags und Verletzungen sind ausgeschlossen, da sie bei zu starker Belastung einfach reißen.

Darüber hinaus setzen die Einrichtungen bei Operationen auf standardisierte Checklisten und die Team-TimeOut-Regelung, eine letzte Sicherheitsstufe vor dem operativen Eingriff, bei der das OP-Team noch einmal alle patientenrelevanten Daten mit Hilfe einer Checkliste überprüft.

www.stiftung-hospital-zum-heiligengeist.de 